\title{
Design and Fabrication of Long Silicon Microring CROWs (Project Report 0642603-Y3)
}

\author{
SHAYAN MOOKHERJEA \\ Department of Electrical and Computer Engineering, University of California, San Diego, MC 0407 La \\ Jolla CA 92093-0407 USA \\ Email:smookher@ucsd.edu
}

\section{Introduction and Goals}

This NSF-funded project [0642603] is a five-year (60 months) CAREER (Faculty Early Career Development Program) unified research and education development program, with a focus on the physics and applications of optical waveguiding in the CROW (Coupled Resonator Optical Waveguide) device structure. The CROW structure is suitable as the foundation of this project because it offers a very high four-wave mixing nonlinearity based on the slowlight effects on each of the pump, signal and idler modes. Since the output depends quadratically on the propagation length (in the low power regime), it is important to realize long CROWs.

\section{Activities}

Research this year (Y3, 02/09 - 01/10) focused on developing designs, fabrication methods and experimental measurement techniques to study CROW structures which comprise coupled silicon microring resonators. Previously, we studied CROWs based on coupled rectangular dielectric microcavities [1], [2]. Longer structures with less disorder can be realized using the coupledmicroring configuration, and this may benefit applications of CROWs as filters and delay lines (optical buffers). Furthermore, based on the free spectral range (FSR) of the constituent microring resonators, the coupled-microring CROW has many transmission passbands within the telecommunications band of wavelengths that span from $1520 \mathrm{~nm}$ to $1600 \mathrm{~nm}$ which are periodically spaced in frequency. This fact can be used in wavelength conversion and correlated photon-pair generation for quantum optics [3].

Models of optical propagation in CROW structures can usually be related to a tight-binding model of propagation [4], [5]. Previously, theoretical studies of nonlinear propagation and wavelength conversion were developed on this basis [6], [7]. However, it was subsequently predicted, and experimentally observed, that propagation in the regions of high dispersion and high nonlinearity would be sensitive to disorder which is inevitable in any real-world fabrication process [8], [2], [9]. It was seen that the enhanced group delay at the band-edges, $\Delta \tau=$ $\tau$ (band-edge) $-\tau$ (band-center), increased linearly with $\mathrm{N}$, and varied from 62 ps $(\mathrm{N}=35)$ to $145 \mathrm{ps}(\mathrm{N}=235)$. Although the ideal CROW theory predicts 
$\Delta \tau \sim \mathrm{N}^{2}$, a linear behavior is predicted by simulation models which we developed for disordered rings, using disorder-emulating scattering matrices which take into account loss and the dispersion measurement resolution.

In collaboration with IBM, silicon microring coupled-resonator optical waveguides (CROWs) with up to 235 unit cells are fabricated and measured. These are the longest CROW structures made, and both amplitude and group delay variation measurements are reported. Fig. 1 shows a typical test site consisting of a bank of 5 CROWs. A total of about 20 such chips have been measured, from two wafers with different roughness reduction strategies, based on our earlier studies into determining what would constitute an optimal strategy for silicon photonic devices. On Wafer 1, a hydrogen annealing step at $900 \mathrm{OC}$ was carried out after the silicon structures were etched. On Wafer 2, a double thermal oxidation step was carried out instead. Averaged transmission over several CROW bands was obtained by using a C-band amplified spontaneous emission source $(+17 \mathrm{dBm}$ power). Linear fits to the spectrallyintegrated power transmitted through CROWs ranging in length from 35 to 235 microrings were obtained for 4 chips from each wafer, and indicate an average loss per ring of $0.20 \mathrm{~dB} /$ ring for Wafer 1 , and $0.062 \mathrm{~dB} /$ ring for Wafer 2 . The lowest insertion (fiber-to-fiber) loss for a 35 ring CROW was $-17 \mathrm{~dB}$.

Group delay measurements were carried out using a single-scan, Jones matrixbased, interferometric spectral measurement instrument (Luna Tech. Optical Vector Analyzer CTe). We have measured record delay-bandwidth products, exceeding 100. However, these are static structures, in which the delay cannot be tuned. Therefore, developing a tuning mechanism for CROWs is a future goal of this program.
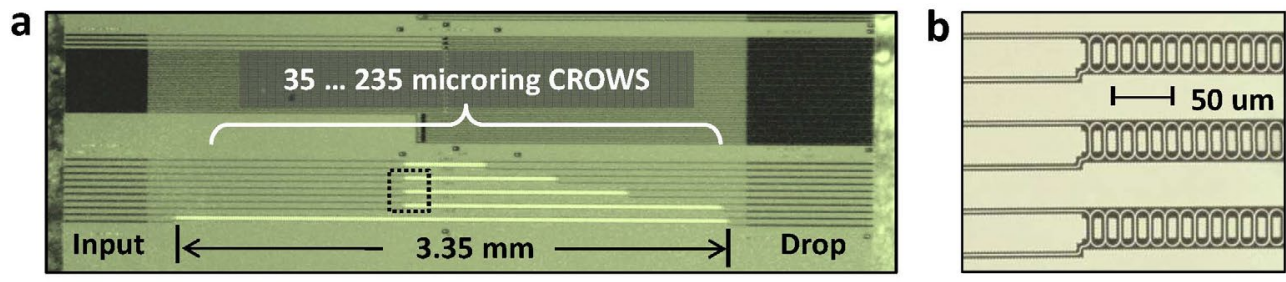

Figure 1 Silicon coupled-microring CROWs ranging from 35 to 235 microrings in a serial array with directional couplers were fabricated using a CMOS compatible process on $200 \mathrm{~mm}$ wafers at IBM and cleaved into $4 \mathrm{~mm}$ long chips. Panel (b) shows in magnification the region indicated by the box in panel (a).

\section{Progress and Impact}

The coupled-microring CROW could be modeled more reliably, and fabricated more easily using conventional (photo-) lithography rather than electron-beam lithography. A collaboration with IBM's silicon photonics group (Dr. W. M. J. Green) provided insights into silicon photonics fabrication and supported technical collaboration on fabricating the CROW structures [10]-[12]. CROWs 
of up to 235 coupled resonators were successfully fabricated and measured, and a good agreement was achieved between experiments and theory [12]-[14].

An accurate knowledge of dispersion is necessary for coupled-microring CROW filters [15] or for potential use of CROWs as compact dispersion compensation devices [16]. We experimentally studied the dispersion of directional couplers using a microring coupled to a waveguide [17]. To help in designing structures in silicon photonics, we developed an extension of coupled-mode theory which is applicable to directional couplers in high index contrast materials, in coupled-waveguide structures [18] and coupled-resonator structures [19]. When the optical power is increased, silicon microring resonators with directional couplers can exhibit interesting behavior, such as bistability and nonlinear on-off switching [20]. However, at lower powers, these effects are not considered to be a significant impairment.

Infrared imaging is a useful experimental tool to study light propagation in CROWs [21], [22]. Dr. Ivan B. Divliansky (Research Scientist, CREOL, Florida) collaborated with us for the electron-beam lithography of coupled microring structures [21]. We demonstrated a non-invasive diagnostic method of quantitative infrared (IR) imaging, applied here to a series cascade of microring resonators fabricated in silicon-on-insulator. The IR images contain information on the otherwise inaccessible individual through-ports and the resonators themselves, providing coupling, loss and intensity-enhancement parameters for the individual rings.
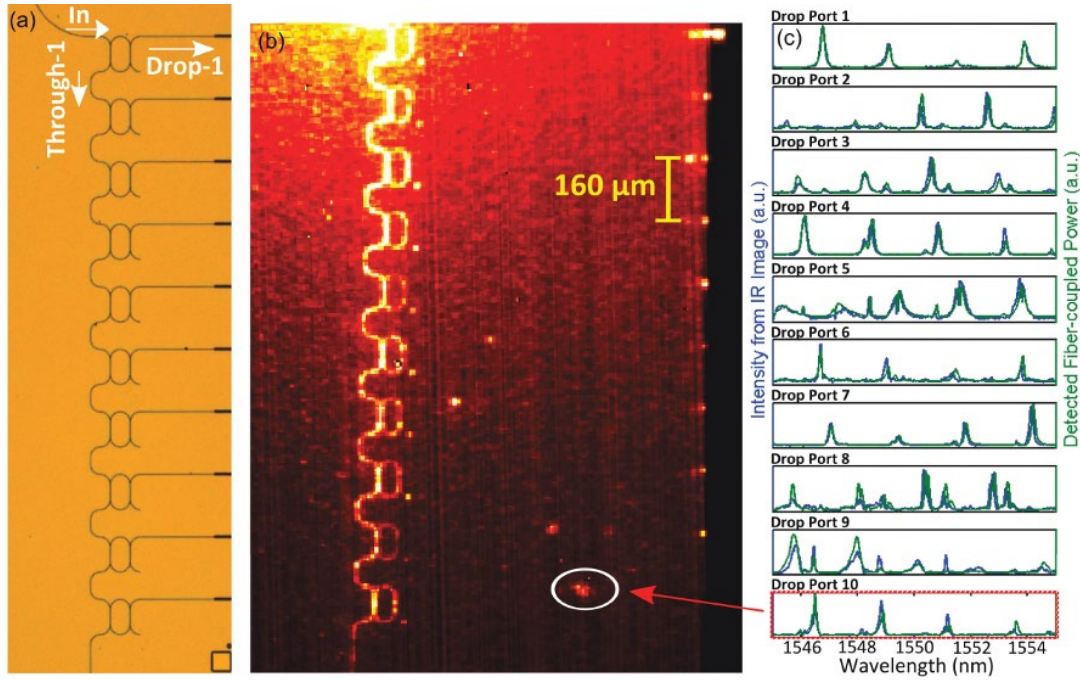

Figure 2 Optical microscope image of 10-ring SCISSOR (radius, $\mathrm{R}=25 \mu \mathrm{m}$, track length, $\mathrm{L}=40$ $\mu \mathrm{m})$. b: IR image of SCISSOR when excited by broadband source. c: Using a tunable laser source, comparison of spectra obtained at the ten drop ports using IR images (blue line) and spectra obtained from detected drop port power using fiber-coupling (green line).

The conventional way of testing an optical waveguide circuit is to couple light into and off the chip using optical fibers, either using lens-tipped small spot- 
size fibers, or conventional fibers with on-chip grating couplers. There are some disadvantages of this approach for multi-resonator optical circuits: firstly, it is usually not possible to measure each individual microring resonator without adding separate input and output waveguides to each resonator. Doing so in a multi-component circuit would add considerably to the footprint and complexity of the structure, and quite likely alter the transfer function substantially. Secondly, inadvertent fabrication defects in the input and output waveguides can prevent light collection from otherwise functional components that lie in the middle of a multi-element circuit.

To overcome these limitations, we developed and demonstrated a non-invasive diagnostic method of quantitative infrared (IR) imaging, which, for example, can be applied to a series cascade of rings, e.g., a side coupled integrated spaced sequence of resonators (SCISSOR) as shown in Fig. 2. We showed the agreement of the drop port transmission spectra obtained using the new approach with the conventional method.

Impact on Human Resources: Two students in the PI's group (one supported directly by this grant and another by an NSF Graduate Fellowship) who have worked on this topic have successfully passed their Candidacy examination and are expected to defend their theses this year. During this year, the PI spent 2 months at the IBM Watson Research Center (July and August, 2009). We initiated a joint study agreement with IBM for 2008-10. We have added a postdoctoral scholar to our research team. Although not directly supported by CAREER funds, the scholar has contributed to the CROW measurements and is a co-author on the relevant papers. One new graduate student is being trained in using advanced lithography equipment at UCSD's Nano3 shared fabrication facility. Equipment that the students are trained on includes electron-beam lithography, reactive ion etcher, inductively-coupled plasma reactive ion etcher, and several other back-end processing tools and processes.

\section{Subsequent Extensions}

Based on the outcomes of this CAREER project, a wide range of subsequent projects were initiated on focused research topics, including nonlinear mixers, harnessing disorder for functionality, and quantum photonics. There are many other areas of nonlinear optics which benefit from a study of chi-(3) optical physics such as spatial soliton formation [23], [24].

The CROW structure can have other applications including generation of quantum light [3], and developing amplifiers and lasers with novel properties [25]. The resonator-enhanced nonlinearity benefits the generation of entangled photon-pair and heralded single-photon generation using silicon photonics [26]. Our initial measurements of spontaneous four-wave mixing (SFWM) were on correlated photon-pair generation and heralded single-photon generation. An 
experimental measurement of entanglement was performed later, using a twophoton interferometer constructed to verify time-energy entanglement [26].

Microring resonators are sensitive to disorder. We studied potential methods for precisely tuning resonators without heaters. Our method is based on fieldinduced local oxidation of $\mathrm{Si}$ to $\mathrm{SiO}_{2}$ via a chemical reaction near an electrically-biased conducting atomic-force microscope tip [27]. Scanning probe lithography has previously been used to modify the resonance frequency of a GaAs photonic crystal cavity. A single silicon microresonator can be monitored and controlled more easily [28] than a long CROW device.

The CROW structures that have been studied so far in this project achieve a high delay-bandwidth product but are passive structures. To tune the (optical group) delay properties in real time, some type of "control knob" is required, which can change the refractive index the constituent materials. Since we use silicon as the waveguide core, a range of options are available. If tuning speed is not a concern, the thermo-optic effect, which we used for tunable filters, is a convenient option [29]. Electro-optic tuning can be implemented in silicon, by introducing $\mathrm{p}$ and $\mathrm{n}$ dopants across the rib waveguide cross section, so that carriers can be injected or removed (depleted) by the application of a voltage. The voltage requirements are low (typically, $<10 \mathrm{~V}$ ) and it is easy to generate the switching waveforms using conventional laboratory equipment [30]. Electro-optic tuning is typically achievable at the nanosecond scale in unoptimized p-n junctions, and faster speeds of about $50-100$ ps $(10 \%-90 \%$ transition time) can potentially be achieved by dopant optimization. Electrooptic tuning in other types of materials can potentially improve tuning speed by another factor of 10 [31]. The CROWs so far have been designed using fullyetched waveguides, and a new design is needed for partially-etched waveguides. A new fabrication process will also be required for the dopants and metallization steps.

\section{Open-Access Reporting Initiative}

PRAISE: This open-access document is provided in support of our PRAISE (Public Report of Activities, Impact and Subsequent Extensions) initiative. What is it? An open-access document shared with the public which describes the research outcomes of publicly-funded projects. For us, these projects are typically funded by the NSF (National Science Foundation).

\section{References}

[1] M. Hammer, "Resonant coupling of dielectric optical waveguides via rectangular microcavities: the coupled guided mode perspective," Optics 
Communications, vol. 214, no. 1-6, pp. 155-170, Dec. 2002, doi: 10.1016/S0030-4018(02)02171-5.

[2] S. Mookherjea, J. S. Park, S.-H. Yang, and P. R. Bandaru, "Localization in silicon nanophotonic slow-light waveguides," Nature Photon, vol. 2, no. 2, pp. 90-93, Feb. 2008, doi: 10.1038/nphoton.2007.278.

[3] J. R. Ong and S. Mookherjea, "Quantum light generation on a silicon chip using waveguides and resonators," Opt. Express, vol. 21, no. 4, p. 5171, Feb. 2013, doi: 10.1364/OE.21.005171.

[4] S. Mookherjea and A. Yariv, "Optical pulse propagation in the tightbinding approximation," Opt. Express, vol. 9, no. 2, p. 91, Jul. 2001, doi: 10.1364/OE.9.000091.

[5] S. Mookherjea and A. Yariv, "Pulse propagation in a coupled resonator optical waveguide to all orders of dispersion," Phys. Rev. E, vol. 65, no. 5, p. 056601, Apr. 2002, doi: 10.1103/PhysRevE.65.056601.

[6] S. Mookherjea and A. Yariv, "Kerr-stabilized super-resonant modes in coupled-resonator optical waveguides," Phys. Rev. E, vol. 66, no. 4, p. 046610, Oct. 2002, doi: 10.1103/PhysRevE.66.046610.

[7] S. Mookherjea and A. Yariv, "Optical pulse propagation and holographic storage in a coupled-resonator optical waveguide," Phys. Rev. E, vol. 64, no. 6, p. 066602, Nov. 2001, doi: 10.1103/PhysRevE.64.066602.

[8] S. Mookherjea, D. S. Cohen, and A. Yariv, "Nonlinear dispersion in a coupled-resonator optical waveguide," Opt. Lett., vol. 27, no. 11, p. 933, Jun. 2002, doi: 10.1364/OL.27.000933.

[9] J. B. Khurgin and R. S. Tucker, Slow light science and applications. Boca Raton: CRC Press, 2009. Accessed: Apr. 29, 2021. [Online]. Available: http://0-marc.crcnetbase.com.fama.us.es/isbn/9781420061529

[10] M. L. Cooper et al., "Waveguide dispersion effects in silicon-oninsulator coupled-resonator optical waveguides," Opt. Lett., vol. 35, no. 18, p. 3030, Sep. 2010, doi: 10.1364/OL.35.003030.

[11] J. R. Ong et al., "Low-power continuous-wave four-wave mixing in silicon coupled-resonator optical waveguides," Opt. Lett., vol. 36, no. 15, pp. 2964-2966, 2011.

[12] M. L. Cooper et al., "235-ring Coupled-Resonator Optical Waveguides," in Conference on Lasers and Electro-Optics 2010, San Jose, California, 2010, p. CTuHH3. doi: 10.1364/CLEO.2010.CTuHH3.

[13] S. Mookherjea and M. A. Schneider, "Avoiding bandwidth collapse in long chains of coupled optical microresonators," Opt. Lett., vol. 36, no. 23, p. 4557, Dec. 2011, doi: 10.1364/OL.36.004557.

[14] M. L. Cooper and S. Mookherjea, "Modeling of Multiband Transmission in Long Silicon Coupled-Resonator Optical Waveguides," IEEE Photon. Technol. Lett., vol. 23, no. 13, pp. 872-874, Jul. 2011, doi: 10.1109/LPT.2011.2141657. 
[15] J. R. Ong, R. Kumar, and S. Mookherjea, "Silicon microring-based wavelength converter with integrated pump and signal suppression," Opt. Lett., vol. 39, no. 15, p. 4439, Aug. 2014, doi: 10.1364/OL.39.004439.

[16] S. Mookherjea, "Using gain to tune the dispersion relation of coupledresonator optical waveguides," IEEE Photon. Technol. Lett., vol. 18, no. 5, pp. 715-717, Mar. 2006, doi: 10.1109/LPT.2006.871144.

[17] R. Aguinaldo, Yiran Shen, and S. Mookherjea, "Large Dispersion of Silicon Directional Couplers Obtained via Wideband Microring Parametric Characterization," IEEE Photon. Technol. Lett., vol. 24, no. 14, pp. 1242-1244, Jul. 2012, doi: 10.1109/LPT.2012.2198639.

[18] M. L. Cooper and S. Mookherjea, "Numerically-assisted coupledmode theory for silicon waveguide couplers and arrayed waveguides," Opt. Express, vol. 17, no. 3, p. 1583, Feb. 2009, doi: 10.1364/OE.17.001583.

[19] S. Mookherjea, "Spectral characteristics of coupled resonators," $J$. Opt. Soc. Am. B, vol. 23, no. 6, p. 1137, Jun. 2006, doi: 10.1364/JOSAB.23.001137.

[20] S. Mookherjea and M. A. Schneider, "The nonlinear microring adddrop filter," Opt. Express, vol. 16, no. 19, p. 15130, Sep. 2008, doi: 10.1364/OE.16.015130.

[21] M. L. Cooper, G. Gupta, J. S. Park, M. A. Schneider, I. B. Divliansky, and S. Mookherjea, "Quantitative infrared imaging of silicon-on-insulator microring resonators," Opt. Lett., vol. 35, no. 5, p. 784, Mar. 2010, doi: 10.1364/OL.35.000784.

[22] S. Mookherjea and H. R. Grant, "High dynamic range microscope infrared imaging of silicon nanophotonic devices," Opt. Lett., vol. 37, no. 22, p. 4705, Nov. 2012, doi: 10.1364/OL.37.004705.

[23] A. Ciattoni, B. Crosignani, S. Mookherjea, and A. Yariv, "Nonparaxial dark solitons in optical Kerr media," Opt. Lett., vol. 30, no. 5, p. 516, Mar. 2005, doi: 10.1364/OL.30.000516.

[24] B. Crosignani, A. Yariv, and S. Mookherjea, "Nonparaxial spatial solitons and propagation-invariant pattern solutions in optical Kerr media," Opt. Lett., vol. 29, no. 11, p. 1254, Jun. 2004, doi: 10.1364/OL.29.001254.

[25] S. Mookherjea, "Semiconductor coupled-resonator optical waveguide laser," Appl. Phys. Lett., vol. 84, no. 17, pp. 3265-3267, Apr. 2004, doi: $10.1063 / 1.1719278$.

[26] R. Kumar, M. Savanier, J. R. Ong, and S. Mookherjea, "Entanglement measurement of a coupled silicon microring photon pair source," Opt. Express, vol. 23, no. 15, p. 19318, Jul. 2015, doi: 10.1364/OE.23.019318.

[27] Y. Shen, I. B. Divliansky, D. N. Basov, and S. Mookherjea, "Perfect set-and-forget alignment of silicon photonic resonators and interferometers," in Optical Fiber Communication Conference/National 
Fiber Optic Engineers Conference 2011, Los Angeles, California, 2011, p. PDPC3. doi: 10.1364/OFC.2011.PDPC3.

[28] M. Savanier, R. Kumar, and S. Mookherjea, "Optimizing photon-pair generation electronically using a $p-i-n$ diode incorporated in a silicon microring resonator," Appl. Phys. Lett., vol. 107, no. 13, p. 131101, Sep. 2015, doi: 10.1063/1.4932047.

[29] R. Aguinaldo et al., "Wideband silicon-photonic thermo-optic switch in a wavelength-division multiplexed ring network," Opt. Express, vol. 22, no. 7, p. 8205, Apr. 2014, doi: 10.1364/OE.22.008205.

[30] S. Mookherjea, J. R. Ong, X. Luo, and L. Guo-Qiang, "Electronic control of optical Anderson localization modes," Nature Nanotech, vol. 9, no. 5, pp. 365-371, May 2014, doi: 10.1038/nnano.2014.53.

[31] X. Wang, P. O. Weigel, J. Zhao, M. Ruesing, and S. Mookherjea, "Achieving beyond-100-GHz large-signal modulation bandwidth in hybrid silicon photonics Mach Zehnder modulators using thin film lithium niobate," APL Photonics, vol. 4, no. 9, p. 096101, Sep. 2019, doi: $10.1063 / 1.5115243$. 